\title{
Diastaphenazine, a new dimeric phenazine from an endophytic Streptomyces diastaticus subsp. ardesiacus
}

\author{
Yiqing $\mathrm{Li}^{1}$, Li Han ${ }^{2}$, He Rong ${ }^{1}$, Liya $\mathrm{Li}^{2}$, Lixing Zhao ${ }^{1}$, Longxia $\mathrm{Wu}^{1}$, Lihua $\mathrm{Xu}^{1}$, Yi Jiang ${ }^{1}$ and Xueshi Huang
}

The Journal of Antibiotics (2015) 68, 210-212; doi:10.1038/ja.2014.124; published online 17 September 2014

Phenazines are a large group of natural and synthesized nitrogencontaining heterocycles, including more than 100 different compounds of natural origin. ${ }^{1}$ The group of phenazine compounds exhibit a broad range of biological activities, such as antibacterial, antimalarial, antitumor and antiparasitic activities. ${ }^{2}$ Natural phenazines are mainly isolated from different microorganisms, including Pseudomonas, Streptomyces and miscellaneous genera. ${ }^{2}$ Even though large numbers of phenazines have been isolated from natural sources, ${ }^{1,3-6}$ the dimeric phenazines were relatively rare. Only esmeraldines $A-B,{ }^{7}$ phenazostatins $\mathrm{A}-\mathrm{D},{ }^{8-11}$ izumiphenazines $\mathrm{A}-\mathrm{B}^{12}$ and phenazinolines $\mathrm{A}-\mathrm{E}^{13}$ were found till now and all of them were isolated from streptomycete. In the course of screening for new bioactive compounds from endophytic actinomycete sources, a new cytotoxic dimeric phenazine, diastaphenazine (1), along with a known compound, izumiphenazine C (2) (Figure 1) was isolated from the fermentation broth of Streptomyces diastaticus subsp. ardesiacus. In this paper, we report the fermentation, isolation, structural elucidation, and cytotoxic and antibacterial activities of 1 .

The producing organism was isolated from surface-sterilized tissue of Artemisia annua collected from Xishuangbanna, Yunnan province, China. The strain was identified as Streptomyces diastaticus subsp. ardesiacus according to the phylogenetic analysis. The genomic DNA of the strain was extracted and used as a template for PCR-mediated amplification of $16 \mathrm{~S}$ ribosomal DNA. The amplicons were used for sequencing and the resulting $16 \mathrm{~S}$ ribosomal DNA sequence was compared with those of the type strains of validly published species in the genus Streptomyces. Phylogenetic analysis showed that the strain shared a higher $16 \mathrm{~S}$ ribosomal RNA gene sequence similarity with the closely related strain S. diastaticus subsp. ardesiacus NRRL B-1773 ${ }^{\mathrm{T}}$ (accession number DQ026631). Meanwhile, the strain was examined for a number of key phenotypic properties known to be of value in streptomycete systematics, and the presence of L, L-diaminopimelic acid in the peptidoglycan together with its colonial characteristics supported its assignment to the genus Streptomyces.
A slant culture of the strain was inoculated into $500 \mathrm{ml}$ Erlenmeyer flasks containing $100 \mathrm{ml}$ of seed medium composed of yeast extract $0.4 \%$, glucose $0.4 \%$, malt extract $0.5 \%$, multiple vitamins solution $0.35 \mathrm{ml}^{-1}, \mathrm{pH} 7.2$ with no adjustment and cultured for 2 days at $28^{\circ} \mathrm{C}$ on a rotary shaker at 220 r.p.m. This seed culture was used to inoculate the fermentation medium with $10 \%$ volume. The fermentation was carried out in $1000 \mathrm{ml}$ Erlenmeyer flasks containing $300 \mathrm{ml}$ of fermentation medium containing starch $2.4 \%$, beef extract $0.3 \%$, glucose $0.1 \%$, yeast extract $0.5 \%$, peptone $0.3 \%, \mathrm{CaCO}_{3} 0.4 \%$ at $\mathrm{pH}$ 7.0 with no adjustment and cultured for 7 days at $28{ }^{\circ} \mathrm{C}$ on a rotary shaker at 220 r.p.m. for upscale fermentation.

The completed fermentation broth (701) was separated into filtrate and mycelium by centrifugation. The culture filtrate was extracted with ethyl acetate and the mycelium was extracted with acetone. After concentrating the ethyl acetate and acetone-soluble portions under reduced pressure, the two portions were combined and dried to yield $54 \mathrm{~g}$ extract. The dried extract was then separated by silica gel column chromatography $\left(\mathrm{CHCl}_{3}-\mathrm{MeOH}\right.$, gradient $\left.40: 1-1: 1(\mathrm{v} / \mathrm{v})\right)$ into seven fractions. Fraction 4 was subjected to gel chromatography on Sephadex LH-20 (Amersham Pharmacia Biotech, Uppsala, Sweden) $(\mathrm{MeOH})$ to produce four fractions. Fraction 4.2 was then purified by silica gel column chromatography $\left(\mathrm{CHCl}_{3}-\mathrm{MeOH} 30: 1\right.$ $(\mathrm{v} / \mathrm{v}))$ and yielded $10 \mathrm{mg}$ of compound 2. Fraction 5 was applied to gel chromatography on Sephadex LH-20 (MeOH) to produce three subfractions. Fraction 5.1 was further separated by silica gel column chromatography $\left(\mathrm{CHCl}_{3}-\mathrm{MeOH} 30: 1(\mathrm{v} / \mathrm{v})\right)$ to yield $60 \mathrm{mg}$ of a semipure material. Additional purification by gel chromatography on Sephadex LH-20 (MeOH) delivered $15 \mathrm{mg}$ of compound 1 .

Compound 1 was obtained as a yellow amorphous powder. $[\alpha]_{\mathrm{D}}^{20}$ -1248 (c 0.11 , dimethyl sulfoxide). UV (MeOH) $\lambda_{\max }(\log \varepsilon) 371$ (3.69), $250(4.26) \mathrm{nm}$. IR (KBr) $\nu_{\max } 3427,1708,1627,1579 \mathrm{~cm}^{-1}$. ESI-MS $m / z 437[\mathrm{M}+\mathrm{H}]^{+}, 459[\mathrm{M}+\mathrm{Na}]^{+}, 895[2 \mathrm{M}+\mathrm{Na}]^{+}, 435$ $[\mathrm{M}-\mathrm{H}]^{-}, 471[\mathrm{M}+\mathrm{Cl}]^{-}, 893[2 \mathrm{M}+\mathrm{Na}-2 \mathrm{H}]^{-}$. High resolution

${ }^{1}$ Key Laboratory of Microbial Diversity in Southwest China, Ministry of Education, Yunnan University, Kunming, P.R. China and ${ }^{2}$ Institute of Microbial Pharmaceuticals, College of Life and Health Sciences, Northeastern University, Shenyang, P.R. China

Correspondence: Dr Y Li, Key Laboratory of Microbial Diversity in Southwest China, Ministry of Education, Yunnan University, Kunming, Yunnan 650091, P.R. China. E-mail: yiqingli@ynu.edu.cn

or Professor X Huang, Institute of Microbial Pharmaceuticals, College of Life and Health Sciences, Northeastern University, Wenhua Road 3-1, Shenyang, Liaoning 110819, P.R. China.

E-mail: huangxs@mail.neu.edu.cn

Received 21 April 2014; revised 8 August 2014; accepted 13 August 2014; published online 17 September 2014 
electron impact-MS $m / z 436.1168$ (calcd for $\mathrm{C}_{25} \mathrm{H}_{16} \mathrm{~N}_{4} \mathrm{O}_{4}, 436.1172$ ). For ${ }^{1} \mathrm{H}$ and ${ }^{13} \mathrm{C}$ NMR data see Table 1.

The molecular formula of 1 was established as $\mathrm{C}_{25} \mathrm{H}_{16} \mathrm{~N}_{4} \mathrm{O}_{4}$ by high resolution electron impact-MS. The IR spectrum of 1 indicated the presence of a hydroxyl group $\left(3427 \mathrm{~cm}^{-1}\right)$, a carboxyl group (1708 $\left.\mathrm{cm}^{-1}\right)$ and aromatic rings $\left(1627,1579 \mathrm{~cm}^{-1}\right)$. The ${ }^{1} \mathrm{H}$ NMR spectrum of 1 (Table 1) showed eight aromatic proton signals at $\delta=8.43(1 \mathrm{H}, \mathrm{d}$, $J=8.0 \mathrm{~Hz}), 8.40(1 \mathrm{H}, \mathrm{d}, J=8.0 \mathrm{~Hz}), 8.11(1 \mathrm{H}, \mathrm{d}, J=8.8 \mathrm{~Hz}), 8.00(1 \mathrm{H}$, t, $J=8.0 \mathrm{~Hz}), 7.93(1 \mathrm{H}, \mathrm{d}, J=8.8 \mathrm{~Hz}), 7.46(1 \mathrm{H}, \mathrm{t}, J=8.0 \mathrm{~Hz}), 7.25$ $(1 \mathrm{H}, \mathrm{d}, J=8.0 \mathrm{~Hz}), 7.05(1 \mathrm{H}, \mathrm{d}, J=8.0 \mathrm{~Hz})$ as well as three methine signals at $\delta=5.06(1 \mathrm{H}, \mathrm{t}, J=4.5 \mathrm{~Hz}), 4.58(1 \mathrm{H}, \mathrm{d}, J=4.5 \mathrm{~Hz})$ and 4.36 $(1 \mathrm{H}$, brt). In addition, a methylene $\mathrm{AB}$ signals appeared at $\delta=3.68$ $(1 \mathrm{H}, \mathrm{dd}, J=17.6,4.9 \mathrm{~Hz})$ and $3.05(1 \mathrm{H}, \mathrm{d}, J=17.6 \mathrm{~Hz})$. The ${ }^{13} \mathrm{C}$ NMR data suggested twenty-five carbons altogether, and the DEPT experiment showed eight aromatic methines $(\delta=133.2,133.0,130.7,129.8$, $128.8,128.6,117.7$ and 111.7), three methines $(\delta=75.0,54.9$ and $40.2)$ and one methylene $(\delta=31.9)$, respectively. Furthermore, thirteen quaternary carbons were presented, including one carboxyl carbon $(\delta=166.7)$ and twelve aromatic quaternary carbons. ${ }^{1} \mathrm{H}$ and ${ }^{13} \mathrm{C}$ NMR data suggested that $\mathbf{1}$ possesses a dimeric phenazine skeleton like those related compounds, phenazinolins A-E. ${ }^{13}$ 2D NMR (HMBC, HSQC, COSY and ROESY) correlations suggested two phenazine structural units $\mathrm{A}$ and $\mathrm{B}$ (Figure 2). The linkage between fragments A and B were determined by HMBC experiments. HMBC correlations were observed between $\mathrm{H}-8 \quad(\delta=7.93)$ and C-9' $(\delta=54.9)$; H-9' $(\delta=4.58)$ and C-6 $(\delta=140.4), \mathrm{C}-7(\delta=147.0), \mathrm{C}-5^{\prime}$ a $(\delta=153.9)$, C-9'a $(\delta=152.0)$; H-7' $(\delta=4.36)$ and C-6 $(\delta=140.4)$, C-7 $(\delta=147.0)$, C-5'a $(\delta=153.9)$ showed C-7 linking with C-9'
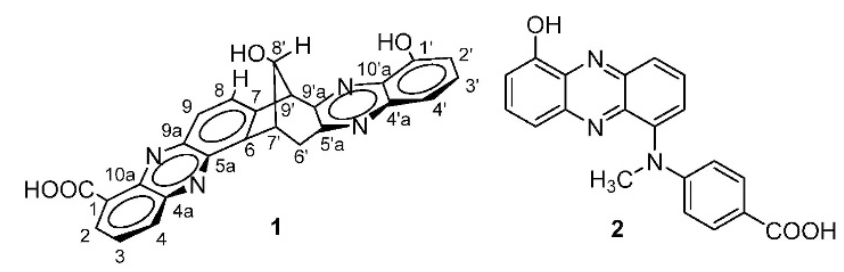

Figure 1 Structures of compounds 1 and 2. $(\delta=54.9)$, and C-6 $(\delta=140.4)$ bonding to C-7' $(\delta=40.2)$ (Figure 2). The planar structure of 1 was also confirmed by ROESY spectrum. NOE correlations between H- $4^{\prime}(\delta=7.25)$ and $\mathrm{H}-6^{\prime} \quad(\delta=3.05$ and $3.68)$ confirmed the hydroxyl group was located at $\mathrm{C}-1^{\prime}$. NOE correlations among $\mathrm{H}-8^{\prime}(\delta=5.06) / \mathrm{H}-6^{\prime} \beta(\delta=3.05)$ determine the relative configurations of $\mathrm{C}-8^{\prime}$. NOE correlations observed between H-8 $(\delta=7.93) / \mathrm{H}-9^{\prime}(\delta=4.58), \mathrm{H}-4(\delta=8.43) / \mathrm{H}-6^{\prime} \beta(\delta=3.05)$ and H-7' $(\delta=4.36)$ supported the connectivities between units A and B. Therefore, the structure of $\mathbf{1}$ was determined (Figure 1) and was named as diastaphenazine considering its microbiological origin $S$. diastaticus subsp. ardesiacus.

The known compound izumiphenazine C (2) was identified by comparison of the MS, ${ }^{1} \mathrm{H}$ and ${ }^{13} \mathrm{C}$ NMR data with the reported spectroscopic data. ${ }^{12}$

To evaluate the cytotoxic activity of $\mathbf{1}$, we measured its cytotoxic effects on five human tumor cell lines including human large-cell lung carcinoma cell line (H460), human hepatocellular liver carcinoma cell line (HepG2), human colon carcinoma cell line (HCT116), human cervix carcinoma cell line (HeLa) and human gastric carcinoma cell line (BGC-823) with an MTT assay procedure. ${ }^{14}$ Compound 1 showed weak cytotoxicity against five human tumor cell lines BGC-823, HeLa, HCT116, HepG2 and H460 with $\mathrm{IC}_{50}$ values of 14.9, 28.8, 65.2, 82.5, and $>100 \mu \mathrm{M}$, respectively. Adriamycin as a positive control showed $\mathrm{IC}_{50}$ values $1.48,0.97,1.38,0.50$ and $0.98 \mu \mathrm{m}$ against BGC-823, HeLa, HCT116, HepG2 and H460 cell lines, respectively.

The antimicrobial effects of $\mathbf{1}$ were also assayed against Staphylococcus aureus (ATCC 25923), Escherichia coli (ATCC 25922), and a

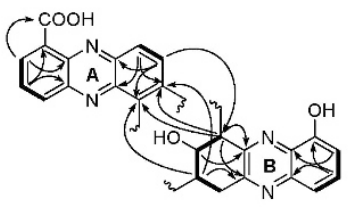

${ }^{1} \mathrm{H}-{ }^{1} \mathrm{H} \operatorname{COSY}-\mathrm{HMBC} \longrightarrow$ b

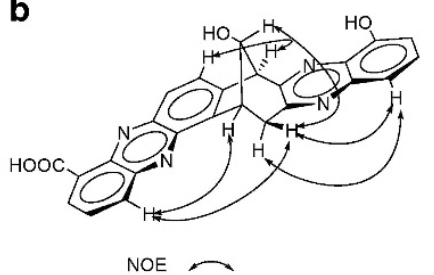

Figure 2 (a) COSY and HMBC correlations of units A and B of compound 1 . (b) Key NOE correlations of 1 .

Table $1{ }^{1} \mathrm{H}\left(600 \mathrm{MHz}\right.$, DMSO- $\left.d_{6}\right)$ and ${ }^{13} \mathrm{C}$ NMR (150 MHz, DMSO- $\left.d_{6}\right)$ data of compound 1

\begin{tabular}{|c|c|c|c|c|c|}
\hline No. & $\delta c$ & $\delta_{H}$ & No. & $\delta c$ & $\delta_{H}$ \\
\hline 1 & 129.8 & - & $1^{\prime}$ & 153.3 & - \\
\hline 2 & 133.2 & $8.40(1 \mathrm{H}, \mathrm{d}, J=8.0 \mathrm{~Hz})$ & $2^{\prime}$ & 111.7 & $7.05(1 \mathrm{H}, \mathrm{d}, J=8.0 \mathrm{~Hz})$ \\
\hline 3 & 130.7 & $8.00(1 \mathrm{H}, \mathrm{t}, J=8.0 \mathrm{~Hz})$ & $3^{\prime}$ & 129.8 & $7.46(1 \mathrm{H}, \mathrm{t}, J=8.0 \mathrm{~Hz})$ \\
\hline 4 & 133.0 & $8.43(1 \mathrm{H}, \mathrm{d}, J=8.0 \mathrm{~Hz})$ & $4^{\prime}$ & 117.7 & $7.25(1 \mathrm{H}, \mathrm{d}, J=8.0 \mathrm{~Hz})$ \\
\hline $4 a$ & 142.4 & - & $4 a^{\prime}$ & 142.3 & - \\
\hline $5 a$ & 139.6 & - & $5 a^{\prime}$ & 153.9 & - \\
\hline 6 & 140.4 & - & $6^{\prime}$ & 31.9 & $\begin{array}{c}\alpha 3.68(1 \mathrm{H}, \mathrm{dd}, J=17.6,4.9 \mathrm{~Hz}) \\
\beta 3.05(1 \mathrm{H}, \mathrm{d}, J=17.6 \mathrm{~Hz})\end{array}$ \\
\hline 7 & 147.7 & - & $7^{\prime}$ & 40.2 & $4.36(1 \mathrm{H}, \mathrm{brt})$ \\
\hline 8 & 128.6 & $7.93(1 \mathrm{H}, \mathrm{d}, J=8.8 \mathrm{~Hz})$ & $8^{\prime}$ & 75.0 & $5.06(1 \mathrm{H}, \mathrm{t}, J=4.5 \mathrm{~Hz})$ \\
\hline 9 & 128.8 & $8.11(1 \mathrm{H}, \mathrm{d}, J=8.8 \mathrm{~Hz})$ & $9^{\prime}$ & 54.9 & $4.58(1 \mathrm{H}, \mathrm{d}, J=4.5 \mathrm{~Hz})$ \\
\hline $9 a$ & 140.7 & - & $9 a^{\prime}$ & 152.0 & - \\
\hline $10 a$ & 139.3 & - & $10 a^{\prime}$ & 131.5 & - \\
\hline \multirow[t]{2}{*}{$1-\mathrm{COOH}$} & 166.7 & - & $1^{\prime}-\mathrm{OH}$ & & $10.33(1 \mathrm{H}, \mathrm{brs})$ \\
\hline & & & $8^{\prime}-\mathrm{OH}$ & & $6.26(1 \mathrm{H}, \mathrm{brs})$ \\
\hline
\end{tabular}

Abbreviation: DMSO, dimethyl sulfoxide. 
Candida albicans (ATCC 10231) using the micro broth dilution method. ${ }^{15} \mathbf{1}$ showed antibacterial activity against $S$. aureus with a MIC value of $64 \mu \mathrm{g} \mathrm{ml}^{-1}$ and was inactive against $E$. coli and $C$. albicans at $128 \mu \mathrm{g} \mathrm{ml}^{-1}$. Ciprofloxacin and amphothericin B were used as positive controls with MIC values $0.5,0.12$ and $0.5 \mu \mathrm{g} \mathrm{ml}^{-1}$ against S. aureus, E. coli and C. albicans, respectively.

\section{ACKNOWLEDGEMENTS}

This work was funded by the National Natural Science Foundation of China (Grant No. 21262041, U0932601), the Yunnan Provincial Natural Science Foundation (Grant No. 2011FB004) and the Basic Scientific Research Fund of the Northeastern University, China (No. N120820002), and supported by the Program for New Century Excellent Talents in University.

1 Cimmino, A. et al. Phenazines and cancer. Nat. Prod. Rep. 29, 487-501 (2012).

2 Laursen, J. B., Nielsen, J. Phenazine natural products: Biosynthesis, synthetic analogues, and biological activity. Chem. Rev. 104, 1663-1685 (2004).

3 Umezawa H., Hayano S., Maeda K., Ogata Y., Okami Y. On a new antibiotic, griseolutein, produced by streptomyces. Jpn Med. J. 1950; 3: 111-117.

4 Geiger, A., Keller-Schierlein, W., Brandl, M., Zähner, H. Metabolites of microorganisms. 247. Phenazines from Streptomyces antibioticus, strain Tu2706. J. Antibiot. 41 1542-1551 (1988).
5 Krastel, P., Zeeck, A., Gebhardt, K., Fiedler, H. P., Rheinheimer, J. Endophenazines $A \sim D$, new phenazine antibiotics from the athropod associated endosymbiont Streptomyces anulatus. II. Structure elucidation. J. Antibiot. 55 801-806 (2002)

6 Fotso, S. et al. Modified phenazines from an Indonesian Streptomyces sp. J. Nat. Prod 73, 472-475 (2010).

7 Keller-Schierlein, W., Geiger, A., Zähner, H., Brandl, M. Metabolites from microorganisms. 251. The esmeraldines A and B, green pigments from Streptomyces antibioticus, strain Tu 2706. Helv. Chim. Acta 71, 2058-2070 (1988).

8 Yun, B. S. et al. Structures of phenazostatins A and B, neuronal cell protecting substances of microbial origin. Tetrahedron Lett. 37, 8529-8530 (1996).

$9 \mathrm{Kim}, \mathrm{W}$. G. et al. New diphenazines with neuronal cell protecting activity, phenazostatins A and B, produced by Streptomyces sp. J. Antibiot. 50, 715-721 (1997).

$10 \mathrm{Kim}$, W. G. et al. Phenazostatin C, a new diphenazine with neuronal cell protecting activity from Streptomyces sp. J. Antibiot. 52, 758-761 (1999).

11 Maskey, R. P., Kock, I., Helmke, E., Laatsch, H. Isolation and structure determination of phenazostatin $D$, a new phenazine from a marine actinomycete isolate Pseudonocardia sp. B6273. Z. Naturforsch.s B 58, 692-694 (2003).

12 Abdelfattah, M. S., Kazufumi, T., Ishibashi, M. Izumiphenazines A-C: isolation and structure elucidation of phenazine derivatives from Streptomyces sp. IFM 11204 J. Nat. Prod. 73, 1999-2002 (2010).

13 Ding, Z. G. et al. Phenazinolins A-E: novel diphenazines from a tin mine tailings-derived Streptomyces species. Org. Biomol. Chem. 9, 2771-2776 (2011).

14 Zheng, D. et al. Isostreptazolin and sannaphenol, two new metabolites from Streptomyces sannanensis. Molecules 17, 836-842 (2012).

15 Zhao, J. L. et al. Antimicrobial metabolites from the endophytic fungus Pichia guilliermondii isolated from Paris polyphylla var. yunnanensis. Molecules 15, 7961-7970 (2010).

Supplementary Information accompanies the paper on The Journal of Antibiotics website (http://www.nature.com/ja) 